Bangladesh J. Bot. 48(1): 187-194, 2019 (March)

\title{
MORPHOLOGICAL MUTANTS OF ZOYSIA JAPONICA STEUD. INDUCED BY GAMMA RAY IRRADIATION
}

\author{
Mohd Abdul Halim Bin Baharun Azahar, Abdul Shukor Juraimi*, Mohd Rafi \\ Yusof ${ }^{1}$, Abdul Rahim Harun ${ }^{2}$, SM Samsuzzaman ${ }^{3}$ AND MD KAMAL UdDIN ${ }^{4}$ \\ Department of Crop Science, Faculty of Agriculture, University Putra Malaysia, \\ Serdang, Selangor, Malaysia
}

Keywords: Gamma ray irradiation, Mutagenesis, Semi-dwarf, Zoysia japonica

\begin{abstract}
Study of Zoysia japonica morphological improvement and breeding by induced mutation using gamma ray irradiation was conducted. Mutagenesis by gamma ray irradiation was employed using $0,20,40,60,80$, 100, 120 and 140 Gy to treat 30 single node stolons per treatment. It was demonstrated that single node stolon is suitable for gamma ray irradiation mutagenesis in Z. japonica. It has been identified that 76 Gy as the value LD50 is effective to induce mutagenesis on $Z$. japonica. Survival rate of $Z$. japonica stolon was greatly reduced when irradiated with higher dosages. This experiment was repeated using LD50 on 1500 single node stolons. Thirty nine morphological mutants were identified and evaluated. Most of the mutants were semi-dwarf and have horizontal growing pattern with reduced internode length and leaf blade length. The altered morphological traits were stable after third cutting back (M1V3) shown by their morphological performance. Mutation breeding is effective in improving $Z$. japonica when easily recognized cultivars are needed.
\end{abstract}

\section{Introduction}

The functions of turfgrasses are to provide beauty and attractiveness for human activities such as in landscape and recreational area (Beard and Green 1994). Turfgrasses can also be used to control water and wind erosion of soil, eliminating dust and mud problems, provide stability to highways, slope and riverbank, and reduce glare, noise air pollution, heat build-up and visual pollution. In Malaysia, among the most valuable warm season turfgrass; mostly used are bermudagrass, zoysiagrass and carpetgrass (Uddin et al. 2009), because turfgrasses still have some weakness that need to be improved. Turfgrass improvement and breeding still in progress of development in Malaysia. Further work is required for investigating genetic variation of useful traits for turfgrass improvement and breeding.

Turfgrass breeding aims at improving the characteristics of plants so that they become more desirable agronomically and economically. Exploitation of natural and induced genetic diversity is the basic requirement of turfgrass breeding in developing plant varieties. Plant breeders are handicapped due to lack of availability or non-existence of desired genotypes and phenotypes. Mutation breeding is relatively a quicker method for improvement of turfgrass. Mutation breeding has been used in recent years as a valuable supplement to the method of plant breeding in the development of better crop cultivars (Awan 1991). The inheritance of important economic traits such as yield, quality, adaptation, pest and stress resistance, upon which much of the future of plant improvement depends, can be understood through the analysis of a wide range of induced

*Author for correspondence: <ashukor@ump.edu.my>. ${ }^{1}$ Institute of Tropical Agriculture, University Putra Malaysia, Serdang, Selangor, Malaysia. ${ }^{2}$ Malaysian Institute of Nuclear Technology Research, Bangi, Selangor, Malaysia, ${ }^{3}$ Soil Resources Development Institute, Krishi Khamar Sarak, Dhaka 1215, ${ }^{4}$ Department of Land Management, Faculty of Agriculture, University Putra Malaysia, Serdang, Selangor, Malaysia. 
mutations. Several workers have attempted for induction of mutation using either physical or chemical mutagens for evolving new genotypes. Issues of enhanced turfgrass improvement depend primarily on increasing new cultivars, product and their maintenance. There are a lot of potential turfgrasses in Malaysia but due to lack of some characteristics, these turfgrasses are not in their top performance. Therefore, the objectives were to determine irradiation mutagenesis of single node cuttings and to identify new semi-dwarf mutants from zoysiagrass (Z. japonica) with good vigour and for cultivar improvement.

\section{Materials and Methods}

The experiment was performed to expose the single node stolon to a different range of dosages gamma ray from $137 \mathrm{Cs}$, that is, $0,20,40,60,80,100,120$ and $140 \mathrm{~Gy}$, to determine the dosages for $\mathrm{LD}_{50}(50 \%$ over control). Stolons were collected randomly from Faculty of Agriculture Turf Plot at Taman Pertanian Universiti Putra Malaysia (UPM) of University Putra Malaysia, Serdang, Selangor and the attached soil was washed off with tap water. The stolons were cut into single node cuttings. Shoots and roots were trimmed to $3-4 \mathrm{~cm}$ in length so that the cuttings would have similar size in irradiation treatment. The cuttings were sealed in plastic bags and irradiated with various dosages of gamma ray, using Biobeam GM 800, Germany with 137Cs source at Gamma Cell Laboratory, Malaysian Institute of Nuclear Technology Research (MINT), Bangi. The control is the cutting which was not irradiated, but kept in plastic bags and sprayed with water to maintain moisture. The cuttings were then planted to plastic trays which consist of $80 \%$ river sand and $20 \%$ peat grow for recovery in the greenhouse at $25 \pm 5^{\circ} \mathrm{C}$. In addition, lime and basic fertilizer phosphorus (P) was used for improving soil $\mathrm{pH}$ and rooting at $1.0 \mathrm{~kg} / 100 \mathrm{~m}^{2}$. Survival and plant height of the stolon was scored 6 weeks later. Survival rate was calculated as the percentage of the number of treated stolons that survived divided by the number of non-treated stolon that survived. Value of $\mathrm{LD}_{50}$ was determined based on survival rate and plant height, by using regression method and the regression type is polynomial. Probit analysis (Finney 1971) was conducted to determine $\mathrm{LD}_{50}$ using SAS program. Regression is a method of fitting a line to data to compare the relationship of the response variable or independent variable (survival rate) that represent $\mathrm{Y}$-axis to the independent variable (gamma ray dosage) that represent $\mathrm{X}$ - axis. For production of numerous mutants, 1500 single node stolons of $Z$. japonica were collected and treated with 76 Gy using Biobeam GM 800, Germany with 137Cs source at MINT. Irradiated stolons were planted into biodegradable tray and maintained for two months in the greenhouse to determine survival rate and to identify mutants. All the surviving mutants were transplanted into plastic pots and isolated using cutting back technique. Parameter leaf length, leaf width, leaf sheath and stem internode length were collected at every stage to test their stability. Design used was completely randomize design (CRD) with four replications. All putative mutants, as determined by morphological changes, were then transferred to a tray for further evaluations after third cutting back. Mutants with consistent morphological changes and growth vigour were further characterized by frequency distribution according to the mutant lines.

Plant height, leaf length, leaf width, leaf sheath and stem internode were measured using Digimatic Caliper (Mitutoyo) and were recorded in millimetre $(\mathrm{mm})$ unit. The numbers of leaves per shoot were calculated manually. The data were measured from three $25 \mathrm{~cm}^{2}$ quadrates which taken in each pot. Ten samples were recorded randomly for three quadrate and the averages were calculated. Shoot density was measured and calculated manually according to the number of shoots in $100 \mathrm{~cm}^{2}$ quadrat. The data were analysed using Statistical Analysis System (SAS Program version 9.0). ANOVA was carried out and compared using Tukey's Studentized Range (HSD) test at $\mathrm{p}<0.05$. 


\section{Results and Discussion}

Irradiated single node stolon of $Z$. japonica reddened within one week after treatment and generally remained dormant for 3 to 4 weeks until either resuming growth or dying. Both survival and plant height were significantly reduced at higher gamma ray dosages (Fig. 1). Coefficient of determination for polynomial regression was higher for survival $\left(r^{2}=0.997\right)$ than for plant height $\left(r^{2}=0.978\right)$.

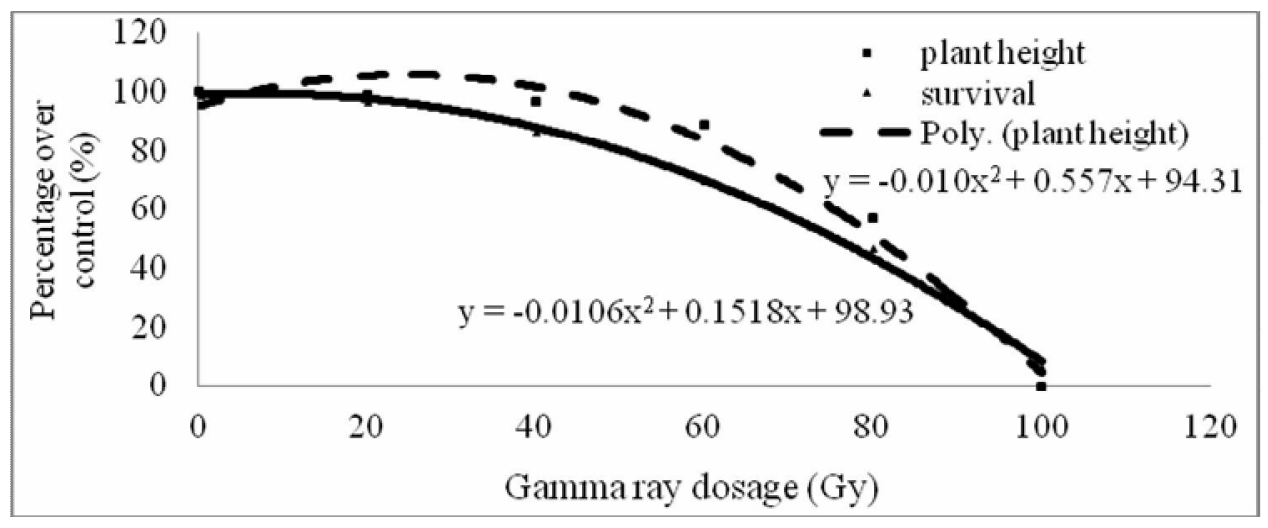

Fig. 1. Effect of gamma ray irradiation on survival and plant height of Z. japonica.

Both responses (survival and plant height) appeared related. Simple transformations such as probit analysis to linearize gamma ray response were tried but were less effective. Logistic curves, including one based on a mechanistic model for unicellular systems were cumbersome and would have required more data points (Haynes 1979). Based on the graph, visual inspection of survival and plant height responses indicate different value of LD50 which are 76 and $80 \mathrm{~Gy}$, respectively. The main purpose of this test is to determine the suitable dose of gamma ray irradiation to be used in the actual experiment. Nevertheless, the dose of 76 Gy was applied as optimum dose based on survival data in the actual experiment which is the one which produces the maximum frequency of mutations and causes the minimum killing.

The highest leaf length at M1V1 population scored with $14.72 \mathrm{~mm}$, followed by $10.48,8.94$ and $7.54 \mathrm{~mm}$. Meanwhile, for M1V2 population, the highest leaf length scored with $10.64 \mathrm{~mm}$, followed by $8.96,8.54$ and $8.14 \mathrm{~mm}$. M1V3 population showed no significant difference with the data 8.06, 7.69, 7.41 and $6.85 \mathrm{~mm}$. The highest leaf width at M1V1 population scored with 2.31 $\mathrm{mm}$, followed by $2.25,2.01$ and $1.74 \mathrm{~mm}$. Meanwhile, for M1V2 population, the highest leaf length scored with $2.34 \mathrm{~mm}$, followed by $2.0,1.78$ and $1.75 \mathrm{~mm}$. M1V3 population showed no significant difference with the data $2.39,2.35,2.26$ and $2.22 \mathrm{~mm}$ ). Leaf sheath were significantly different at M1V1 and M1V2 population on Z13-1-4 mutant line (Fig. 2). The highest at M1V1 population scored with $8.50 \mathrm{~mm}$, followed by $8.43,5.56$ and $4.55 \mathrm{~mm}$. Meanwhile, for M1V2 population, the highest leaf length scored with $8.44 \mathrm{~mm}$, followed by $5.50,5.30$ and $4.82 \mathrm{~mm}$. M1V3 population showed no significant different with the data 5.68, 5.59, 5.31 and $4.87 \mathrm{~mm}$. Stem internode length was significantly different at M1V1 and M1V2 population on Z13-1-4 mutant line (Fig. 2). The highest at M1V1 population scored with $13.11 \mathrm{~mm}$, followed by 10.30 , 6.46 and $5.22 \mathrm{~mm}$. For M1V2 population, the highest leaf length scored with $10.29 \mathrm{~mm}$, followed by $6.10,5.42$ and $5.10 \mathrm{~mm}$. M1V3 population showed no significant difference with the data 6.12 , $5.91,5.66$ and $5.41 \mathrm{~mm}$. 

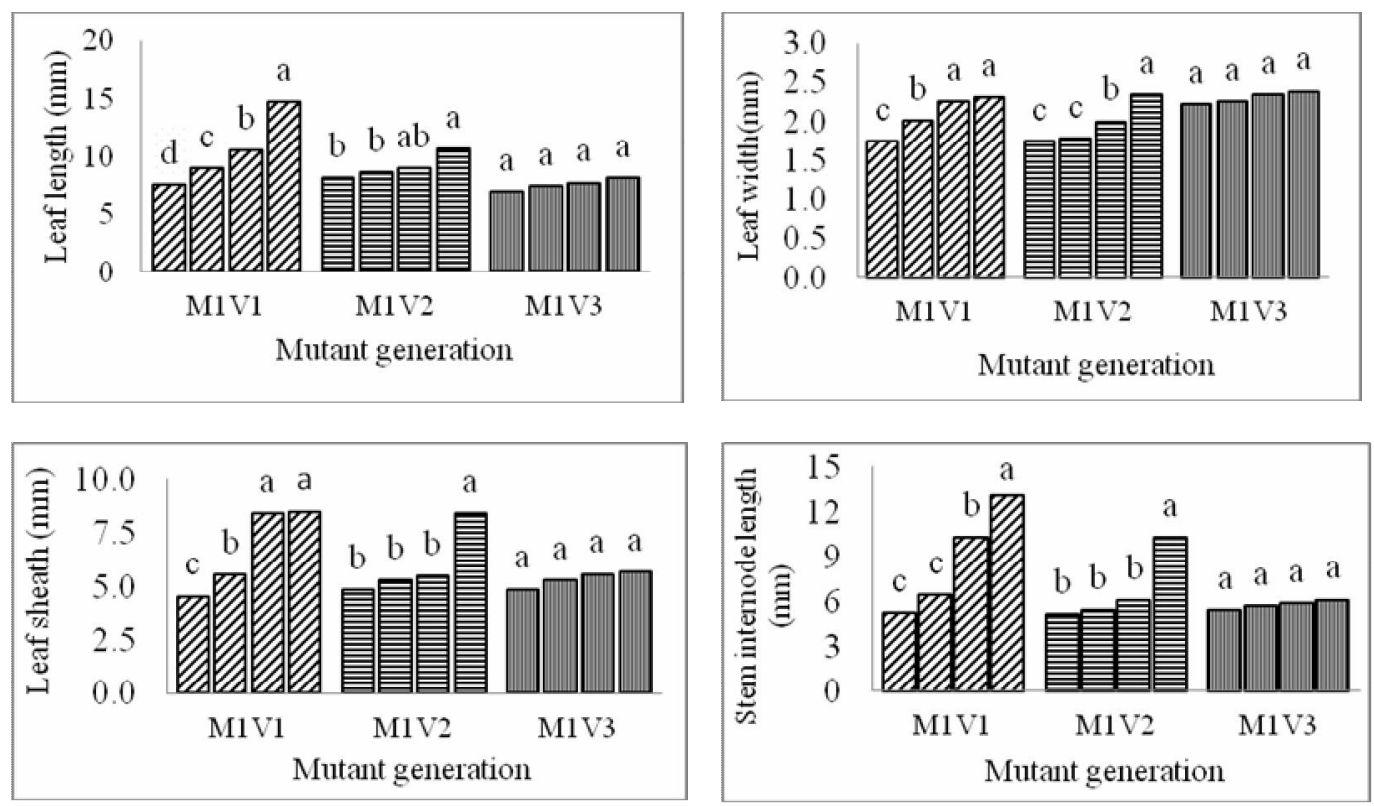

Fig. 2. Multiple bar diagram showing leaf length, leaf width, leaf sheath and stem internode variation at different stage of mutant generation for Z13-1-4 mutant line.

Mean plant height of treated plants $(18.24 \mathrm{~mm})$ showed significant difference at $(\mathrm{p}<0.05)$ as compared to the control $(18.40 \mathrm{~mm})$. The frequency distribution of plant height ranging from 7.5 $\mathrm{mm}$ to $42.5 \mathrm{~mm}$ for treated and $12.5 \mathrm{~mm}$ to $27.5 \mathrm{~mm}$ for control plants, respectively. Gamma ray irradiation provided wider frequency distribution for Z. japonica. This gives greater opportunity to select semi-dwarf type mutants and other desirable morphological (Fig. 3). Mean leaf length of treated plants $(15.98 \mathrm{~mm})$ showed no significant difference at $(\mathrm{p}<0.05)$ as compared to the control $(15.94 \mathrm{~mm})$. The frequency distribution of leaf length ranging from $7.5 \mathrm{~mm}$ to $37.5 \mathrm{~mm}$ for treated and 7.5 to $27.5 \mathrm{~mm}$ for control plants, respectively. Gamma ray irradiation provided wider frequency distribution for Z. japonica. Mean leaf width of treated plants $(2.09 \mathrm{~mm})$ showed no significant difference at $(\mathrm{p}<0.05)$ as compared to the control $(2.03 \mathrm{~mm})$.

The frequency distribution of leaf width ranging from $0.75 \mathrm{~mm}$ to $3.75 \mathrm{~mm}$ for treated and $1.25 \mathrm{~mm}$ to $2.75 \mathrm{~mm}$ for control plants, respectively. Gamma ray irradiation provided wider frequency distribution for $Z$. japonica. This gives greater opportunity to select finer leaf texture mutants. Mean shoot density of treated plants (141.12) showed no significant difference at $(\mathrm{p}<$ $0.05)$ as compared to the control (131.94). The frequency distribution of shoot density ranging from 115.0 to $175.0 \mathrm{~mm}$ for treated and 115.0 to $145.0 \mathrm{~mm}$ for control plants, respectively. Gamma ray irradiation significantly altered the number of shoot density for Z. japonica. This gives more choice to select more desirable and attractive grass with higher shoot density (Fig. 3).

Gamma ray irradiation significantly altered morphological characters, more specific according to each mutant line (Tables 1 and 2). Most of them were recovered from single node stolon irradiated at $76 \mathrm{~Gy}$, produce semi-dwarf types. Three of them were remarkable as the best semi dwarf-type growths which are C131-3-1, C59-2-2 and C85-1-2. The radiosensitivity test is required in order to determine the value of lethality dosage of LD50 (50\% over control) for $Z$. japonica. The study showed that increasing the dose of mutagen can cause greater toxicity or in 
other words, with only slightly low dosage can cause lethality (Hahn and Soyer 2008). Based on Michelle (1994), the lethal dose $\mathrm{LD}_{50}$ was calculated following regression of the survival data per cent lethality over control on gamma rays doses. However, another regression parameter also can be used for radiosensitivity test. From the experiment, two different parameters, i.e. survival rate and plant height were recorded and analysed for radiosensitivity test. Data from plant height and the value of $\mathrm{LD}_{50}$ can be used for producing semi-dwarf type turfgrass in future. Anon. (1997) reported that the suitable dose should be between $20 \%$ lower and $20 \%$ higher from the midpoint of the estimated desirable dose range by the laboratory test. Nevertheless, the dose of 76 Gy was applied as optimum dose based on survival data in the actual experiment which is the one which produces the maximum frequency of mutations and causes the minimum killing. It was found that irradiated $Z$. japonica stolons exhibit some obvious variation like dwarfism, reduce length of internode and leaves texture in M1V1 population. Ghazali et al. (2003) reported that variations observed among M1V1 population were not solely due to genetic mutation, instead it could be
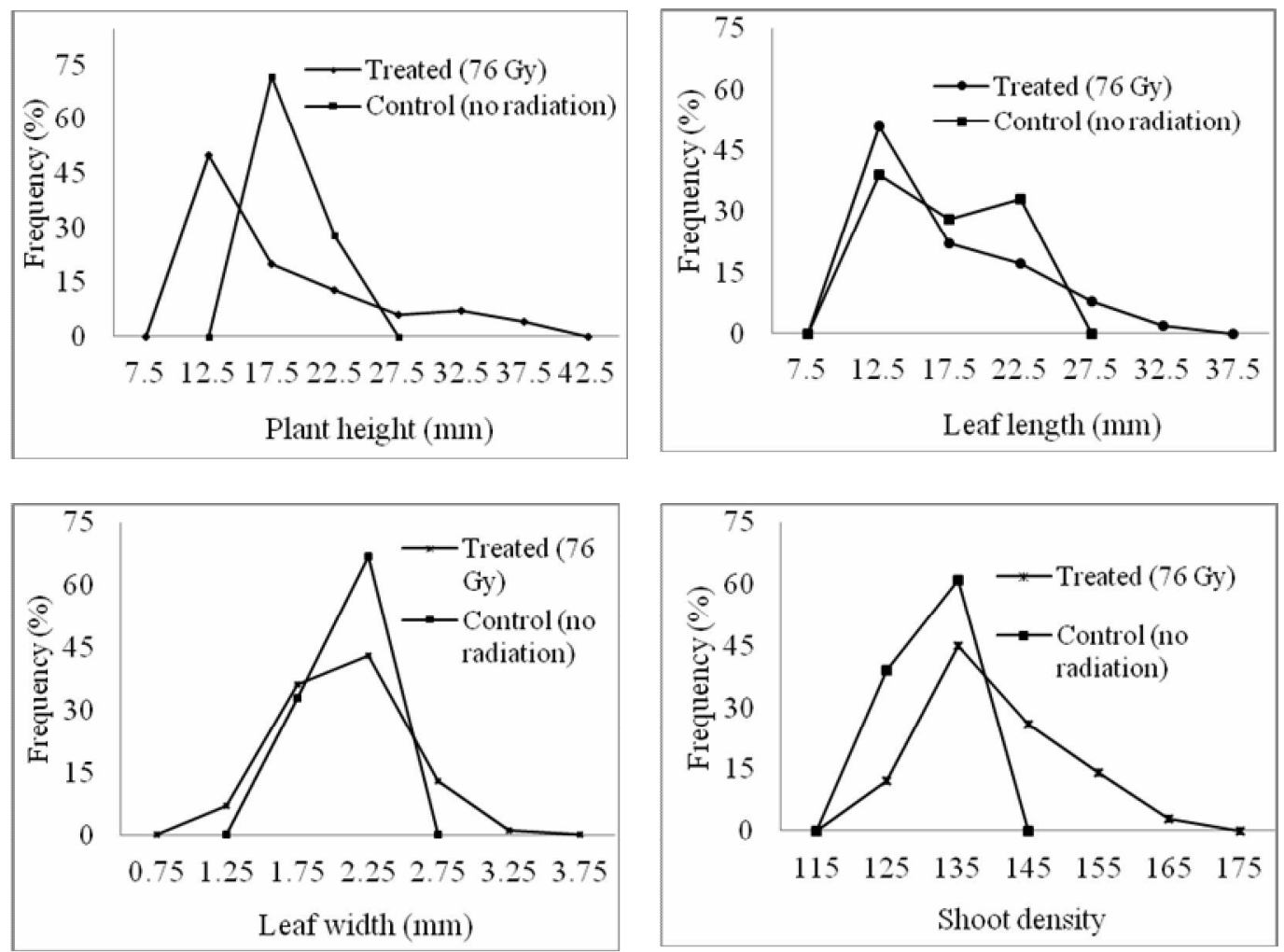

Fig. 3. Frequency distribution for plant height, leaf length, leaf width and shoot density.

cause by somatic mutation. The mutant cell will continue to divide and the individual will contain a patch of tissue of genotype different from the cell of the rest of the body, initiate somatic mutation to occur (Anon. 2002). According to Lineberger (1996), this effect called chimeras where cells of more than one genotype (genetic makeup) are found growing adjacent in the tissue of the plant. The most common types of chimeras can be seen through is variegated plant as the basic example presenting basic concept. Usually, most of them cannot be inherited to the next generation and disappear. From this study, this effect actually shown by mutant line of Z13-1-4. 
Table 1. Comparison of plant height (PH), leaf length (LL), leaf width (LW), sheath length (SL), stem internode length (SeTL) and stolon internode length (SoTL) of the selected mutant lines and the control $\left(\mathbf{Z}_{0}\right)$.

\begin{tabular}{|c|c|c|c|c|c|c|}
\hline Mutant lines & $\mathrm{PH}(\mathrm{mm})$ & LL (mm) & $\mathrm{LW}(\mathrm{mm})$ & $\mathrm{SL}(\mathrm{mm})$ & $\operatorname{SeTL}(\mathrm{mm})$ & SoTL(mm) \\
\hline $\mathrm{Z} 0$ & $14.72 \mathrm{e}-\mathrm{g}$ & $20.06 \mathrm{a}-\mathrm{f}$ & $2.14 \mathrm{f}-\mathrm{k}$ & $8.43 \mathrm{a}-\mathrm{c}$ & $10.29 \mathrm{f}-\mathrm{i}$ & $11.06 \mathrm{f}-\mathrm{i}$ \\
\hline Z1-2-1 & $14.58 \mathrm{e}-\mathrm{g}$ & $20.03 \mathrm{a}-\mathrm{f}$ & $2.24 \mathrm{~d}-\mathrm{i}$ & $6.66 \mathrm{e}-\mathrm{i}$ & $9.56 \mathrm{i}-\mathrm{k}$ & $9.96 \mathrm{i}-1$ \\
\hline Z2-2-1 & $8.66 \mathrm{fg}$ & 9.97 g-i & $1.75 \mathrm{~m}-\mathrm{o}$ & $3.73 \mathrm{o}-\mathrm{r}$ & $9.67 \mathrm{ij}$ & $10.01 \mathrm{~h}-1$ \\
\hline Z4-2-1 & $14.58 \mathrm{e}-\mathrm{g}$ & $16.05 \mathrm{c}-\mathrm{h}$ & $1.61 \mathrm{op}$ & $7.05 \mathrm{~d}-\mathrm{g}$ & $10.76 \mathrm{f}-\mathrm{h}$ & $11.02 \mathrm{f}-\mathrm{i}$ \\
\hline Z6-2-2 & $10.48 \mathrm{fg}$ & $10.51 \mathrm{~g}-\mathrm{i}$ & $2.00 \mathrm{i}-\mathrm{n}$ & $5.61 \mathrm{i}-\mathrm{k}$ & $13.22 \mathrm{c}$ & $13.41 \mathrm{~cd}$ \\
\hline Z8-4-1 & $29.33 \mathrm{~b}-\mathrm{d}$ & $12.84 \mathrm{f}-\mathrm{i}$ & $3.01 \mathrm{a}$ & $8.82 \mathrm{a}$ & $12.86 \mathrm{~cd}$ & $13.21 \mathrm{~cd}$ \\
\hline Z9-2-4 & $32.73 \mathrm{a}-\mathrm{c}$ & $16.71 \mathrm{c}-\mathrm{h}$ & $1.98 \mathrm{i}-\mathrm{n}$ & $5.44 \mathrm{j}-1$ & $5.01 \mathrm{st}$ & $5.48 \mathrm{q}-\mathrm{s}$ \\
\hline Z9-3-3 & $32.64 \mathrm{a}-\mathrm{c}$ & $22.94 \mathrm{a}-\mathrm{d}$ & $2.00 \mathrm{i}-\mathrm{n}$ & $7.49 \mathrm{~b}-\mathrm{e}$ & $5.32 \mathrm{r}-\mathrm{t}$ & $5.77 \mathrm{q}-\mathrm{s}$ \\
\hline Z13-2-1 & $7.59 \mathrm{fg}$ & $10.36 \mathrm{~g}-\mathrm{i}$ & $1.92 \mathrm{j}-\mathrm{n}$ & $2.35 \mathrm{~s}$ & $8.12 \mathrm{~m}-\mathrm{o}$ & $8.74 \mathrm{mn}$ \\
\hline Z13-1-4 & $8.51 \mathrm{fg}$ & $7.58 \mathrm{hi}$ & $2.31 \mathrm{c}-\mathrm{h}$ & $4.351-0$ & $5.18 \mathrm{r}-\mathrm{t}$ & $5.24 \mathrm{rs}$ \\
\hline Z14-1-3 & $18.28 \mathrm{~d}-\mathrm{g}$ & $17.55 \mathrm{~b}-\mathrm{g}$ & $1.40 \mathrm{pq}$ & $5.43 \mathrm{j}-1$ & $8.92 \mathrm{j}-\mathrm{n}$ & $9.33 \mathrm{j}-\mathrm{m}$ \\
\hline Z16-3-2 & $15.62 \mathrm{e}-\mathrm{g}$ & $16.86 \mathrm{c}-\mathrm{h}$ & $2.17 \mathrm{f}-\mathrm{k}$ & $6.71 \mathrm{e}-\mathrm{i}$ & $8.94 j-n$ & 9.14 k-m \\
\hline Z17-3-1 & $15.03 \mathrm{e}-\mathrm{g}$ & $16.16 \mathrm{c}-\mathrm{h}$ & $2.00 \mathrm{i}-\mathrm{n}$ & $5.09 \mathrm{j}-\mathrm{m}$ & $4.85 \mathrm{t}$ & $4.94 \mathrm{~s}$ \\
\hline Z18-2-4 & 30.46 a-d & $22.21 \mathrm{a}-\mathrm{e}$ & $2.51 \mathrm{~b}-\mathrm{e}$ & $7.05 \mathrm{~d}-\mathrm{g}$ & $17.28 \mathrm{a}$ & $18.62 \mathrm{a}$ \\
\hline Z19-2-2 & $35.60 \mathrm{a}-\mathrm{c}$ & $20.50 \mathrm{a}-\mathrm{f}$ & $2.17 \mathrm{f}-\mathrm{k}$ & $6.72 \mathrm{~d}-\mathrm{i}$ & $8.06 \mathrm{~m}-\mathrm{o}$ & $8.74 \mathrm{mn}$ \\
\hline Z24-1-2 & $9.86 \mathrm{fg}$ & $12.75 \mathrm{f}-\mathrm{i}$ & $2.14 \mathrm{f}-\mathrm{k}$ & $8.61 \mathrm{ab}$ & $13.45 \mathrm{c}$ & $14.07 \mathrm{c}$ \\
\hline Z26-3-1 & $8.94 \mathrm{fg}$ & $13.34 \mathrm{e}-\mathrm{i}$ & $2.17 \mathrm{f}-\mathrm{k}$ & $2.83 \mathrm{q}-\mathrm{s}$ & 7.42 op & 7.96 no \\
\hline Z29-3-3 & $10.02 \mathrm{fg}$ & $10.22 \mathrm{~g}-\mathrm{i}$ & $1.88 \mathrm{k}-\mathrm{o}$ & $4.01 \mathrm{~m}-\mathrm{p}$ & $6.02 \mathrm{q}-\mathrm{s}$ & $6.56 \mathrm{pq}$ \\
\hline Z31-1-1 & $9.01 \mathrm{fg}$ & $12.33 \mathrm{f}-\mathrm{i}$ & $1.831-\mathrm{o}$ & $3.12 \mathrm{p}-\mathrm{s}$ & $10.94 \mathrm{e}-\mathrm{g}$ & $11.13 \mathrm{f}-\mathrm{h}$ \\
\hline Z36-3-1 & $10.58 \mathrm{fg}$ & $13.11 \mathrm{e}-\mathrm{i}$ & $2.08 \mathrm{~g}-1$ & $3.91 \mathrm{n}-\mathrm{r}$ & $9.32 \mathrm{i}-1$ & $9.71 \mathrm{j}-\mathrm{m}$ \\
\hline Z39-3-1 & $34.06 \mathrm{a}-\mathrm{c}$ & $20.54 \mathrm{a}-\mathrm{f}$ & $2.39 \mathrm{c}-\mathrm{f}$ & $7.33 \mathrm{c}-\mathrm{f}$ & $8.241-\mathrm{o}$ & $8.79 \mathrm{mn}$ \\
\hline Z41-2-1 & $18.40 \mathrm{~d}-\mathrm{f}$ & $11.65 \mathrm{f}-\mathrm{i}$ & $1.811-\mathrm{o}$ & $6.80 \mathrm{~d}-\mathrm{h}$ & $11.16 \mathrm{ef}$ & $11.43 \mathrm{e}-\mathrm{g}$ \\
\hline Z44-2-4 & $15.57 \mathrm{e}-\mathrm{g}$ & $13.80 \mathrm{~d}-\mathrm{i}$ & $1.61 \mathrm{op}$ & $6.12 \mathrm{~g}-\mathrm{k}$ & $8.96 \mathrm{j}-\mathrm{n}$ & $9.02 \mathrm{k}-\mathrm{n}$ \\
\hline Z49-2-4 & $32.52 \mathrm{a}-\mathrm{c}$ & 22.28 a-e & 2.02 h-n & $6.17 \mathrm{f}-\mathrm{i}$ & $6.00 \mathrm{q}-\mathrm{s}$ & $6.11 \mathrm{p}-\mathrm{r}$ \\
\hline $\mathrm{Z} 50-2-3$ & $14.79 \mathrm{e}-\mathrm{g}$ & $19.04 \mathrm{a}-\mathrm{g}$ & $2.54 \mathrm{~b}-\mathrm{d}$ & $7.12 \mathrm{~d}-\mathrm{g}$ & $9.87 \mathrm{~g}-\mathrm{j}$ & 10.14 h-k \\
\hline Z53-3-2 & $8.94 \mathrm{fg}$ & $11.34 \mathrm{f}-\mathrm{i}$ & $1.75 \mathrm{~m}-$ & $2.79 \mathrm{rs}$ & 8.37 1-o & $8.74 \mathrm{mn}$ \\
\hline Z70-1-1 & $14.99 \mathrm{e}-\mathrm{g}$ & $23.06 \mathrm{a}-\mathrm{d}$ & $2.22 \mathrm{e}-\mathrm{j}$ & $6.22 \mathrm{f}-\mathrm{i}$ & $6.75 \mathrm{pq}$ & 6.14 p-r \\
\hline Z75-4-1 & $10.42 \mathrm{fg}$ & $16.10 \mathrm{c}-\mathrm{h}$ & 2.02 h-m & $4.01 \mathrm{~m}-\mathrm{p}$ & 7.88 no & 7.97 no \\
\hline Z80-2-2 & $37.69 \mathrm{ab}$ & $24.62 \mathrm{a}-\mathrm{c}$ & $2.60 \mathrm{bc}$ & $5.66 \mathrm{~h}-\mathrm{k}$ & $9.89 \mathrm{f}-\mathrm{j}$ & $10.33 \mathrm{~g}-\mathrm{j}$ \\
\hline Z81-1-1 & $36.09 \mathrm{a}-\mathrm{c}$ & $27.15 \mathrm{a}$ & $2.78 \mathrm{ab}$ & $8.92 \mathrm{a}$ & $16.97 \mathrm{ab}$ & $17.54 \mathrm{a}$ \\
\hline Z81-2-3 & $24.59 \mathrm{c}-\mathrm{e}$ & $14.82 \mathrm{~d}-\mathrm{i}$ & $2.31 \mathrm{c}-\mathrm{h}$ & $3.48 \mathrm{o}-\mathrm{s}$ & $9.01 \mathrm{j}-\mathrm{m}$ & $9.33 \mathrm{j}-\mathrm{m}$ \\
\hline Z82-2-2 & $16.57 \mathrm{e}-\mathrm{g}$ & $19.29 \mathrm{a}-\mathrm{g}$ & $2.60 \mathrm{bc}$ & $5.00 \mathrm{k}-\mathrm{n}$ & $6.17 \mathrm{qr}$ & $7.00 \mathrm{op}$ \\
\hline Z84-2-3 & $10.46 \mathrm{fg}$ & $12.73 \mathrm{f}-\mathrm{i}$ & $2.10 \mathrm{f}-1$ & 3.76 o-r & 9.76 h-j & $10.11 \mathrm{~h}-\mathrm{k}$ \\
\hline Z91-2-1 & $42.12 \mathrm{a}$ & $26.80 \mathrm{ab}$ & $2.51 \mathrm{~b}-\mathrm{e}$ & $3.99 \mathrm{~m}-\mathrm{q}$ & $9.02 \mathrm{j}-\mathrm{m}$ & $9.22 \mathrm{j}-\mathrm{m}$ \\
\hline Z102-2-4 & $10.58 \mathrm{fg}$ & $14.10 \mathrm{~d}-\mathrm{i}$ & $1.89 \mathrm{k}-\mathrm{o}$ & $7.32 \mathrm{c}-\mathrm{f}$ & 9.79 h-j & $10.11 \mathrm{~h}-\mathrm{k}$ \\
\hline Z104-3-4 & $16.26 \mathrm{e}-\mathrm{g}$ & $15.05 \mathrm{~d}-\mathrm{i}$ & $1.71 \mathrm{no}$ & $8.83 \mathrm{a}$ & $11.89 \mathrm{de}$ & $12.33 \mathrm{de}$ \\
\hline Z116-2-4 & $8.83 \mathrm{fg}$ & $18.02 \mathrm{a}-\mathrm{g}$ & $2.35 \mathrm{c}-\mathrm{g}$ & $3.40 \mathrm{o}-\mathrm{s}$ & $12.89 \mathrm{~cd}$ & $13.26 \mathrm{~cd}$ \\
\hline Z118-2-3 & $11.00 \mathrm{fg}$ & $11.87 \mathrm{f}-\mathrm{i}$ & $2.14 \mathrm{f}-\mathrm{k}$ & $3.48 \mathrm{o}-\mathrm{s}$ & 8.57 k-n & $8.971-n$ \\
\hline Z131-3-1 & $5.77 \mathrm{~g}$ & $6.41 \mathrm{i}$ & $1.12 \mathrm{q}$ & $3.24 \mathrm{o}-\mathrm{s}$ & 10.99 ef & $11.54 \mathrm{ef}$ \\
\hline Z141-1-1 & 19.43 d-f & $12.35 \mathrm{f}-\mathrm{i}$ & $1.73 \mathrm{~m}-\mathrm{o}$ & $7.88 \mathrm{a}-\mathrm{d}$ & $15.98 \mathrm{~b}$ & $16.22 \mathrm{~b}$ \\
\hline
\end{tabular}


Table 2. Comparison of stems thickness (SeT) and stolon thickness (SoT), shoot density (SD), grass color (GR) and number of leaves per shoot (NOL) of the selected mutant lines and the control $\left(\mathbf{Z}_{\mathbf{0}}\right)$.

\begin{tabular}{|c|c|c|c|c|c|}
\hline Mutant lines & $\operatorname{SeT}(\mathrm{mm})$ & SoT(mm) & SD & GR & NOL \\
\hline $\mathrm{ZO}$ & $0.92 \mathrm{c}-\mathrm{g}$ & $0.84 \mathrm{ab}$ & $125.00 \mathrm{~s}$ & $5.69 \mathrm{~cd}$ & $5.25 \mathrm{c}-\mathrm{f}$ \\
\hline Z1-2-1 & $0.89 \mathrm{e}-\mathrm{h}$ & $0.83 \mathrm{ab}$ & $135.50 \mathrm{~m}-\mathrm{o}$ & $5.79 \mathrm{a}-\mathrm{d}$ & $6.75 \mathrm{ab}$ \\
\hline Z2-2-1 & $1.15 \mathrm{~b}$ & $1.01 \mathrm{ab}$ & $128.25 \mathrm{q}-\mathrm{s}$ & $5.81 \mathrm{a}-\mathrm{d}$ & $6.00 \mathrm{a}-\mathrm{e}$ \\
\hline Z4-2-1 & $0.67 \mathrm{~lm}$ & $0.66 \mathrm{~b}$ & $127.25 \mathrm{rs}$ & $5.69 \mathrm{~b}-\mathrm{d}$ & $6.00 \mathrm{a}-\mathrm{e}$ \\
\hline Z6-2-2 & $0.76 \mathrm{~d}-\mathrm{h}$ & $0.86 \mathrm{ab}$ & $138.50 \mathrm{j}-\mathrm{m}$ & $5.79 \mathrm{a}-\mathrm{d}$ & $5.75 \mathrm{a}-\mathrm{e}$ \\
\hline Z8-4-1 & $0.70 \mathrm{k}-\mathrm{m}$ & $0.67 \mathrm{~b}$ & 129.50 p-r & $5.83 \mathrm{a}-\mathrm{d}$ & $5.50 \mathrm{~b}-\mathrm{f}$ \\
\hline Z9-2-4 & $0.71 \mathrm{k}-\mathrm{m}$ & $0.69 \mathrm{~b}$ & 141.50 h-j & $5.84 \mathrm{a}-\mathrm{d}$ & $5.75 \mathrm{a}-\mathrm{e}$ \\
\hline Z9-3-3 & $0.67 \mathrm{~lm}$ & $0.61 \mathrm{~b}$ & $146.50 \mathrm{e}-\mathrm{g}$ & $5.92 \mathrm{a}-\mathrm{c}$ & $5.50 \mathrm{~b}-\mathrm{f}$ \\
\hline Z13-2-1 & 0.89 e-h & $0.86 \mathrm{ab}$ & $136.501-n$ & $5.84 \mathrm{a}-\mathrm{d}$ & $5.75 \mathrm{a}-\mathrm{e}$ \\
\hline Z13-1-4 & $0.76 \mathrm{i}-1$ & $0.72 \mathrm{ab}$ & $154.00 \mathrm{~b}$ & $5.61 \mathrm{~d}$ & 4.75 ef \\
\hline Z14-1-3 & 0.69 k-m & $0.67 \mathrm{~b}$ & $139.25 \mathrm{i}-\mathrm{m}$ & $5.79 \mathrm{a}-\mathrm{d}$ & $5.00 \mathrm{~d}-\mathrm{f}$ \\
\hline Z16-3-2 & 0.80 h-k & $0.77 \mathrm{ab}$ & 133.75 n-o & $5.78 \mathrm{a}-\mathrm{d}$ & $6.75 \mathrm{ab}$ \\
\hline Z17-3-1 & 0.99 c-e & $0.76 \mathrm{ab}$ & $152.50 \mathrm{~b}$ & $5.85 \mathrm{a}-\mathrm{d}$ & $6.75 \mathrm{ab}$ \\
\hline Z18-2-4 & 0.69 k-m & $0.66 \mathrm{~b}$ & $135.50 \mathrm{~m}-\mathrm{o}$ & $5.75 \mathrm{a}-\mathrm{d}$ & $5.25 c-f$ \\
\hline Z19-2-2 & 0.79 h-k & $0.71 \mathrm{ab}$ & $144.00 \mathrm{f}-\mathrm{h}$ & $5.84 \mathrm{a}-\mathrm{d}$ & 4.75 ef \\
\hline Z24-1-2 & $1.19 \mathrm{ab}$ & $1.12 \mathrm{ab}$ & $133.00 \mathrm{n}-\mathrm{p}$ & $5.84 \mathrm{a}-\mathrm{d}$ & $6.00 \mathrm{a}-\mathrm{e}$ \\
\hline Z26-3-1 & $1.03 \mathrm{c}$ & $0.99 \mathrm{ab}$ & $147.75 \mathrm{~d}-\mathrm{f}$ & $5.79 \mathrm{a}-\mathrm{d}$ & $6.00 \mathrm{a}-\mathrm{e}$ \\
\hline Z29-3-3 & $0.91 \mathrm{~d}-\mathrm{g}$ & $0.88 \mathrm{ab}$ & 140.75 h-k & $5.76 \mathrm{a}-\mathrm{d}$ & $5.25 \mathrm{c}-\mathrm{f}$ \\
\hline Z31-1-1 & $0.70 \mathrm{k}-\mathrm{m}$ & $0.68 \mathrm{~b}$ & $148.25 \mathrm{c}-\mathrm{e}$ & $5.79 \mathrm{a}-\mathrm{d}$ & $5.75 \mathrm{a}-\mathrm{e}$ \\
\hline Z36-3-1 & $1.03 \mathrm{c}$ & $0.97 \mathrm{ab}$ & $139.00 \mathrm{j}-\mathrm{m}$ & $5.82 \mathrm{a}-\mathrm{d}$ & 4.75 ef \\
\hline Z39-3-1 & $0.97 \mathrm{c}-\mathrm{f}$ & $0.92 \mathrm{ab}$ & $152.00 \mathrm{bc}$ & $5.86 \mathrm{a}-\mathrm{d}$ & $5.00 \mathrm{~d}-\mathrm{f}$ \\
\hline Z41-2-1 & $0.60 \mathrm{~m}$ & $0.59 \mathrm{~b}$ & $152.00 \mathrm{bc}$ & $5.81 \mathrm{a}-\mathrm{d}$ & $5.75 \mathrm{a}-\mathrm{e}$ \\
\hline Z44-2-4 & 0.79 h-k & $0.72 \mathrm{ab}$ & $128.25 \mathrm{q}-\mathrm{s}$ & $5.78 \mathrm{a}-\mathrm{d}$ & 6.00 a-e \\
\hline Z49-2-4 & $0.74 \mathrm{j}-1$ & $0.70 \mathrm{ab}$ & $153.75 \mathrm{~b}$ & $5.89 \mathrm{a}-\mathrm{c}$ & $6.75 \mathrm{ab}$ \\
\hline Z50-2-3 & $0.97 \mathrm{c}-\mathrm{f}$ & $0.92 \mathrm{ab}$ & 134.00 no & $5.76 \mathrm{a}-\mathrm{d}$ & $5.00 \mathrm{~d}-\mathrm{f}$ \\
\hline Z53-3-2 & $1.29 \mathrm{a}$ & $1.15 \mathrm{ab}$ & $143.25 \mathrm{~g}-\mathrm{I}$ & $5.83 \mathrm{a}-\mathrm{d}$ & $5.25 \mathrm{c}-\mathrm{f}$ \\
\hline Z70-1-1 & 0.89 e-h & $0.81 \mathrm{ab}$ & $151.50 \mathrm{~b}-\mathrm{d}$ & $5.86 \mathrm{a}-\mathrm{c}$ & $6.50 a-c$ \\
\hline Z75-4-1 & $0.82 \mathrm{~g}-\mathrm{j}$ & $0.81 \mathrm{ab}$ & $136.251-n$ & $5.84 \mathrm{a}-\mathrm{d}$ & $5.75 \mathrm{a}-\mathrm{e}$ \\
\hline Z80-2-2 & $0.66 \mathrm{~lm}$ & $0.62 \mathrm{~b}$ & $127.25 \mathrm{rs}$ & $5.79 \mathrm{a}-\mathrm{d}$ & $6.00 \mathrm{a}-\mathrm{e}$ \\
\hline Z81-1-1 & $0.96 \mathrm{c}-\mathrm{f}$ & $0.92 \mathrm{ab}$ & $132.00 \mathrm{o}-\mathrm{q}$ & $5.71 \mathrm{~b}-\mathrm{d}$ & $7.00 \mathrm{a}$ \\
\hline Z81-2-3 & $1.01 \mathrm{~cd}$ & $0.96 \mathrm{ab}$ & $136.75 \mathrm{k}-\mathrm{n}$ & $5.87 \mathrm{a}-\mathrm{c}$ & $6.75 \mathrm{ab}$ \\
\hline Z82-2-2 & $0.70 \mathrm{k}-\mathrm{m}$ & $2.27 \mathrm{a}$ & $144.00 \mathrm{f}-\mathrm{h}$ & $5.89 \mathrm{a}-\mathrm{c}$ & $6.00 \mathrm{a}-\mathrm{e}$ \\
\hline Z84-2-3 & 0.89 e-h & $0.87 \mathrm{ab}$ & $136.501-n$ & $5.97 \mathrm{a}$ & $7.00 \mathrm{a}$ \\
\hline Z91-2-1 & 0.79 h-k & $0.75 \mathrm{ab}$ & 140.25 h-1 & $5.78 \mathrm{a}-\mathrm{d}$ & 4.75 ef \\
\hline Z102-2-4 & $0.87 \mathrm{f}-\mathrm{i}$ & $0.80 \mathrm{ab}$ & $138.50 \mathrm{j}-\mathrm{m}$ & $5.86 \mathrm{a}-\mathrm{c}$ & $4.25 \mathrm{f}$ \\
\hline Z104-3-4 & $0.99 \mathrm{c}-\mathrm{e}$ & $0.97 \mathrm{ab}$ & $136.251-n$ & $5.78 \mathrm{a}-\mathrm{d}$ & $5.25 \mathrm{c}-\mathrm{f}$ \\
\hline Z116-2-4 & $1.17 \mathrm{~b}$ & $1.11 \mathrm{ab}$ & $141.75 \mathrm{~h}-\mathrm{j}$ & $5.93 \mathrm{ab}$ & $6.25 \mathrm{a}-\mathrm{d}$ \\
\hline Z118-2-3 & $1.17 \mathrm{~b}$ & $1.10 \mathrm{ab}$ & $146.00 \mathrm{e}-\mathrm{g}$ & $5.88 \mathrm{a}-\mathrm{c}$ & 4.75 ef \\
\hline Z131-3-1 & 0.69 k-m & $0.64 \mathrm{~b}$ & $182.50 \mathrm{a}$ & $5.86 \mathrm{a}-\mathrm{c}$ & $5.00 \mathrm{~d}-\mathrm{f}$ \\
\hline Z141-1-1 & 0.77 i-1 & $0.72 \mathrm{ab}$ & $139.75 \mathrm{i}-1$ & $5.82 \mathrm{a}-\mathrm{d}$ & $5.00 \mathrm{~d}-\mathrm{f}$ \\
\hline
\end{tabular}


However, mutant variation seems stable after M1V3 population and could not be differentiated statistically. This statement strongly supported by MINT (1998), whereas mutant purification and selection can be done at or after M1V3 population because only a little chimera effect exist or no longer exist. Mutant pure line determination is a must in order to avoid any variation for each $Z$. japonica mutant lines. In some cases, unstable mutation occurred because plant will undergo recombination process during meiosis. As the result, many mutants change the chromosome back to normal and no variation occurred.

These studies demonstrate that single node stolon is suitable for gamma ray irradiation mutagenesis in $Z$. japonica. It identified that $76 \mathrm{~Gy}$ as the value LD50 is effective to induced mutagenesis on Z. japonica. Mutants were identified with significant morphological changes from the treated nodes and the changes were stable after M1V3 cutting back. Most of the mutants have character of semi-dwarf. Thirty nine mutants were obtained from 1500 node cuttings treated at 76 Gy, giving a rate of $2.6 \%$. A large number of single node stolons should be treated as starting material to increase the probability of obtaining higher number of useful mutants. The mutants obtained from this study have great potential to develop into a new cultivar of $Z$. japonica for sports field and for landscaping purpose. However, further studies need to be taken up to test their genetic materials and physiological responses.

\section{References}

Anonimous 2002. The Bio Space Glossary. BIOSPACE. http: / biospace.com/gls. Accessed on 12 Feb. 2013.

Anonimous 1977. Manual on mutation breeding. Technical reports series N0 119. IAEA Vienna, 1977. pp. 169-192.

Awan MA 1991. Use of induced mutations for crop improvement in Pakistan. Int. symposium on the contribution of plant mutation breeding to crop improvement. Vienna Austria. IAEA. 553: 67-72.

Beard JB and Green RL 1994. The role of turfgrasses in environmental protection and their benefits to humans. J. Environ. Qual. 23: 1-16.

Finney DJ 1971. Probit Analysis, 3rd edn. Cambridge University Press, Cambridge, UK.

Ghazali HA, Harun R and Samsudin S 2003. Study on mutagenesis of signal grassBy gamma irradiation. Malaysian Institute for Nuclear Technology Research.

GRIN (Germplasm Resources Information Network) Species Records of Zoysia. Germplasm Resources Information Network. United States Department of Agriculture, Retrieved 2011-03-03.

Hahn ED and Soyer R 2008. Probit and logic model. Differences in a multivariaterealm.

Haynes RH and Eckardt F 1979. Analysis of dose-response patterns in mutation research. Can. J. Genet. Cytol. 21: 277-302.

Lineberger DR 1996. Origin, development and propagation of chimeras. http:/aggie-horticulture.tamu. edu/tissult/chimeralec/chimeras.html. Accessed on 10 November 2012.

Michelle MS, Thompson RD and Weil CS 1994. Computer programme for calculation of median effective dose (LD50) using the method of moving average interpolation. Arch. Tovicol. 68: 332-337.

MINT (Malaysian Institute of Nuclear Technology Research) 1998. Mutation Breeding Manual. The Use of Induced Mutation for Plant improvement in Malaysia, National Committee on the Use of Induced Mutation in Plant Breeding.Malaysian Institute for NuclearTechnology Research, Bangi, Malaysia.

Reynolds WC, Li R, Silva K, Bruneau AH and Qu R 2009. Field performance of mutant and somaclonal variation lines of St. Augustinegrass [Stenotaphrum secundatum (Walt.) Kuntze]. Intl. Turfgrass Soc. Res. J. 11: 573-582.

Uddin MK, Juraimi AS, Ismail MR, Radziah O and Rahim AA 2009. Growth response of eight tropical turfgrass species to salinity. African Journal of Biotechnology 21: 5799-5806. 